\title{
EXPERIMENTAL VALIDATION FOR TR-UWB SYSTEMS By Time Delayed Sampling \& Correlation (TDSC)
}

\author{
Jorge A. Pardiñas-Mir, Muriel Muller \\ Institut Telecom, Telecom SudParis, 9, rue Charles Fourier, 91011 Evry France \\ jorge.pardinas@it-sudparis.eu,muriel.muller@it-sudparis.eu \\ Roger Lamberti, Claude Gimenes \\ Institut Telecom, Telecom SudParis, 9, rue Charles Fourier, 91011 Evry France \\ roger.lamberti@it-sudparis.eu,claudemonique.gimenes@orange.fr
}

\begin{abstract}
Keywords: $\quad$ transmitted reference (TR-UWB), ultra wide band (UWB), CMOS circuit, impulse radio (IR).
Abstract: Detection results of transmitted reference ultra-wideband signals (TR-UWB) are presented. The signals are received through the implementation in CMOS technology of the 'Time Delayed Sampling and Correlation' (TDSC) detection method, which allows the test of its performance and validates the first stage of the synchronization process. This method has been proposed to achieve a UWB system with low cost, low complexity and low power consumption for medium to low data rate applications such as ranging or localization. Detection of such signals has been done successfully in both a direct cable connection as well in a wireless system with a real channel. In both cases they were used in the sub GHz band (group1) and also in the low band UWB signals.
\end{abstract}

\section{INTRODUCTION}

Since the new regulations adopted by the U.S. Federal Communications Commission (F.C.C., 2002) related to the use of the ultra-wideband (UWB) signals, research and industrial work in this area has increased in the last years, leading to the definition of many standards (IEEE, 2007), (ECMA, 2008) and encouraged new applications (Gezici, 2008), (Jofre et al., 2009).

One objective to achieve at the low-data rate communication applications is that of developing an UWB device that has a low complexity, that is low power consuming and that has a low cost. The 'Time Delayed Sampling and Correlation' (TDSC) detection method has been proposed to conceive a receiver that meets such an objective (Muller et al., 2008).

The TDSC detection method is a pseudocoherent receiver based on the correlation between two waveforms captured from a transmitted reference UWB (TR-UWB). The theoretical study of the design parameters for a CMOS implementation of the method using $0.35 \mu \mathrm{m}$ technology, the prototype design and simulation, the characterization and first tests of the prototype were presented in (Hirata-Flores et al., 2008), (Hirata-Flores, 2008) and (Saber et al, 2008).

Work is done for developing a ranging and positioning strategy based on the TDSC detection method considering that UWB signals are well suited for this application because of its time resolution (Gezici et al., 2005).

This paper propose to go ahead with the test platform based on the first validation step and to validate the detection method as the base of the synchronization procedure proposed in (Saber et al, 2008) using the TR-UWB signals acquired thanks to the platform. The TDSC detection method and the integrated circuit that implements it are described briefly in section II. The synchronization process proposed for a receiver that uses the TDSC detection is explained in section III. Section IV presents the platform that has been developed for testing the circuit and the synchronization method along with the results of the detection tests. 


\section{TIME DELAYED SAMPLING AND CORRELATION DETECTION}

\subsection{The TDSC Detection Method}

The TDSC method is based on the use of a UWB signal with a transmitted reference which is called a TR-UWB signal. In its simplest way this signal uses a pair of pulses, called doublet $d(t)$, conforming to the FCC spectrum mask for each symbol transmitted with a period of $\mathrm{T}_{\mathrm{S}}$ seconds: a reference pulse $g_{I}(t)$ and an information pulse $g_{2}(t)$ delayed by $T_{D}$ seconds from the reference and modulated by the information bits $b_{k}$.

$$
\begin{gathered}
. d(t)=g_{1}(t)+g_{2}(t) \\
g_{1}(t)=p(t) \quad g_{2}(t)=b_{k} p\left(t-T_{D}\right)
\end{gathered}
$$

In the case of synchronization or ranging related symbols, $g_{2}(t)$ is not modulated, $b_{k}=1$, carrying the same pulse shape as that of $g_{l}(t)$ but delayed by $\mathrm{T}_{\mathrm{D}}$ seconds.

$$
\begin{gathered}
g_{1}(t)=p(t) \quad g_{2}(t)=p\left(t-T_{D}\right) \\
d(t)=p(t)+p\left(t-T_{D}\right)
\end{gathered}
$$

Figure 1 shows the structure of a TR-UWB symbol for the case where a BPSK modulation is used.

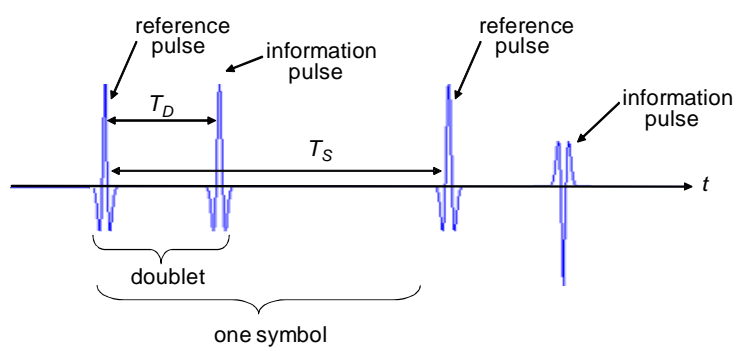

Figure 1: Symbol structure of a TR-UWB signal.

The detection of the TR-UWB signal is made through a correlation receiver which correlates the information pulse with the transmitted reference that has been delayed in time. The delay line must have a wide frequency response, highly linear phase, very good impedance matching and highly stable delay, which is difficult to integrate. The block diagram of such a receiver is shown in figure 2 where the effects of channel and noise have been omitted for ease of illustration.

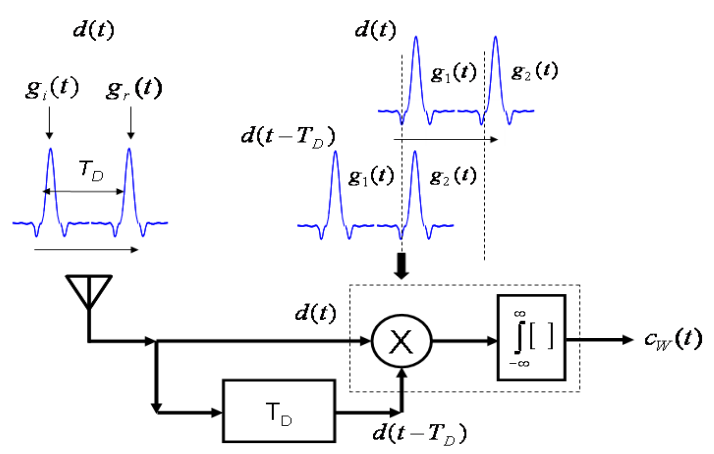

Figure 2: Block diagram of the TR-UWB receiver.

The TDSC detection method proposes the substitution of the wide band analog delay line by a pair of samplers controlled by a digital delay (corresponding to $\mathrm{T}_{\mathrm{D}}$ ). In this way both pulses $g_{I}(t)$ and $g_{2}(t)$ can be sampled and be available at the same time inside the sampling windows. The waveform correlation can be made either using an analog multiplier or by a digital processor. Figure 3 shows the blocks that make the function of time delaying and correlation.

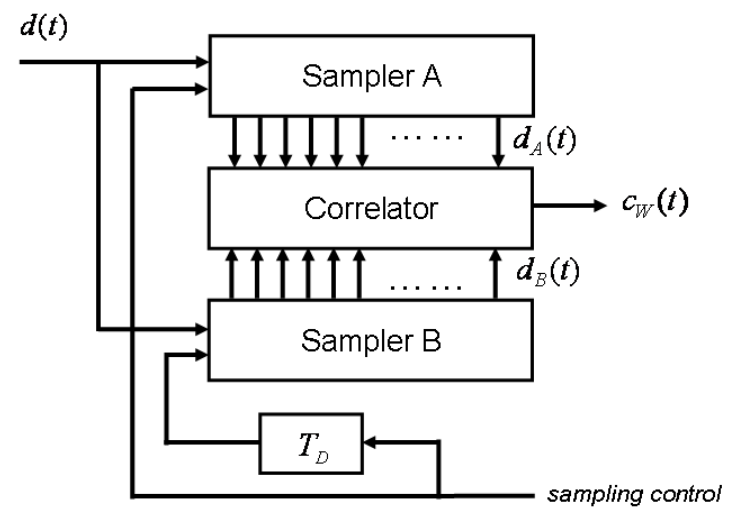

Figure 3: Samplers that substitute the analog delay line.

The value obtained at the output of the correlator is evaluated to determine the value carried by the information pulse. In the example shown, a positive value would mean an information pulse in phase, while a negative value would mean an information pulse with a $180^{\circ}$ phase.

The method was validated according to the IEEE UWB channel model (Molisch et al., 2006) and implemented (Hirata-Flores, 2008) using a $0.35 \mu \mathrm{m}$ CMOS technology. The most important details of this prototype are explained in the next section. 


\subsection{CMOS Implementation}

The block diagram of the prototype circuit that implements the TDSC detection method is shown in figure 4. It includes the two samplers described in the previous section, designed using nMOS transmission gates (TG), each one including a register with a length of 128 cells. The high speed sample clock generation circuit uses as its basis an analogically-adjustable edge triggered asynchronous delay line which allows multi-gigahertz sampling frequencies as high as $7.5 \mathrm{GHz}$. A coplanar wave guide (CPW) is used for guiding the RF signal.

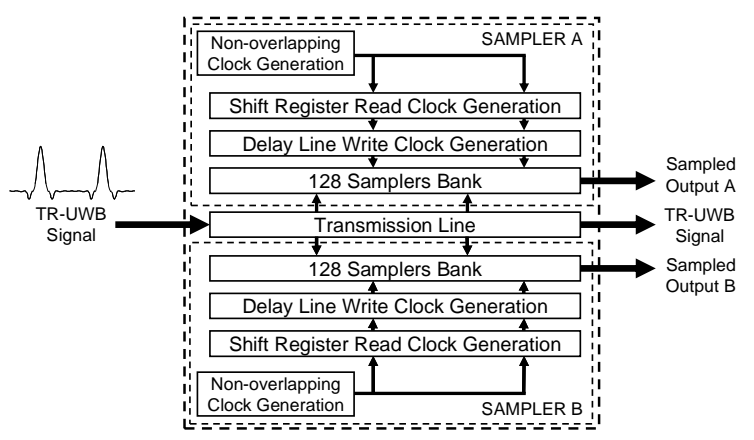

Figure 4: Block diagram of the TDSC prototype circuit.

Assuming that the receiver is synchronized, the contents of this registers, $d_{A 0}$ and $d_{B 0}$, can be expressed as:

$$
\begin{gathered}
\mathbf{d}_{A 0}=\left[\begin{array}{c}
r\left(t_{0}\right) \ldots r\left(t_{0}+n T_{e}\right) \\
\ldots r\left(t_{0}+\left(N_{W}-1\right) T_{e}\right)
\end{array}\right] \\
\mathbf{d}_{B 0}=\left[\begin{array}{c}
r\left(t_{0}+T_{D}\right) \ldots r\left(t_{0}+n T_{e}+T_{D}\right) \\
\ldots r\left(t_{0}+\left(N_{W}-1\right) T_{e}+T_{D}\right)
\end{array}\right] \\
T_{e}=\frac{1}{f_{s}} N_{W}=\frac{T_{W}}{T_{e}}
\end{gathered}
$$

Figure 5 shows the relation between the sampling control pulses and the sampling and recording of each TR-UWB pulse into each of the registers of the TDSC chip, where $T_{W}$ is the length of the register, and time instant $t_{0}$ is the start control for the sampler A.

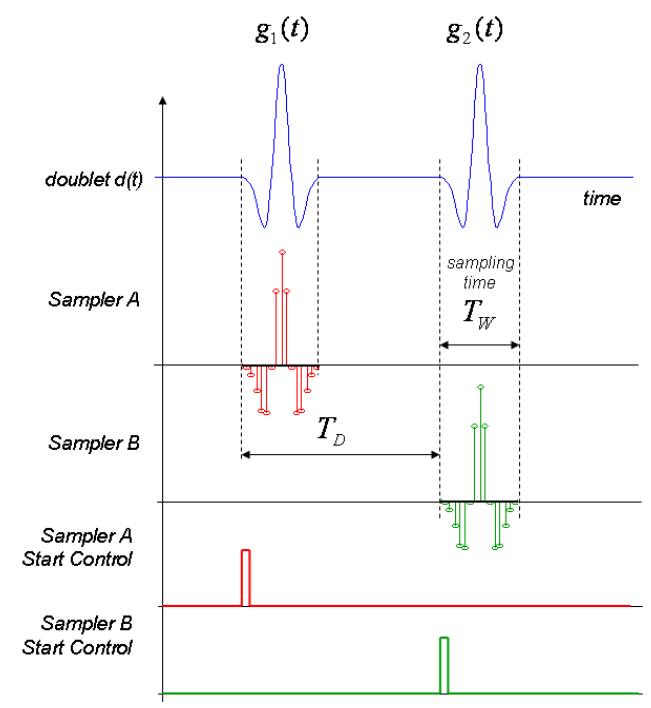

Figure 5: Sampling and recording of each TR-UWB pulse according to control pulses for each separate register.

In figure 6 it is shown the packaged circuit of the TDSC receiver circuit, whose chip dimensions are $\mathrm{L}=3.5 \mathrm{~mm}$ and $\mathrm{W}=1.7 \mathrm{~mm}$.

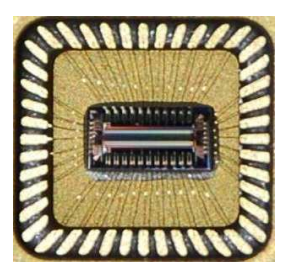

Figure 6: Picture of the TDSC circuit chip.

For the circuit to be able to correctly detect the incoming pulses, a previous synchronization process must be carried out. The next section briefly describes this process.

\section{SYNCHRONIZATION PROCESS}

The goal of the synchronization process is to find a time reference into the symbol for sampling the pulses at the correct time and then be able to detect the information. The technique used here for the TDSC detection method is lightly modified compared to the one proposed in (Saber et al, 2008) in order to manage the worst case where one correlator is used and only one correlation is computed for each symbol. 
The synchronization process is based on the search of the maximum correlation value along the symbol.

The synchronization process starts by sampling the synchronization sequence at a certain reference point $t_{0}$. Pulse waveforms are sampled and saved in the registers $A$ and $B$ of length $T_{w}$ each other. Then the receiver tests the existence of the pulses into the sampled windows by correlating them according to (8). The computed value corresponds to the maximum value of their cross-correlation.

$$
\begin{aligned}
c_{W 0} & =\sum_{n=0}^{N_{W}-1} d\left(t_{0}+n T_{e}\right) \cdot d\left(t_{0}+n T_{e}+T_{D}\right) \\
& =\mathbf{d}_{A 0}^{T} \cdot \mathbf{d}_{B 0}
\end{aligned}
$$

The resulting $c_{W 0}$ value is saved and other correlations are made and saved, applying a time $\Delta$ shift along the whole symbol. When all correlations, as described in (9), are obtained, the maximum value corresponds to the presence of the pulses into the registers.

$$
\begin{gathered}
c_{W k}=\sum_{n=0}^{N_{W}-1}\left[\begin{array}{c}
d\left(t_{0}+k \Delta+n T_{e}\right) \\
\cdot d\left(t_{0}+k \Delta+n T_{e}+T_{D}\right)
\end{array}\right] \\
c_{W}=\left\lfloor c_{W 0}, c_{W 1}, \ldots c_{W k} \ldots, c_{W(K-1)}\right\rfloor \\
k=0,1,2, \ldots, K-1
\end{gathered}
$$

where $\mathrm{K}$ is the number of needed correlations in order to sweep the whole symbol.

$$
K=\frac{T_{S}}{\Delta}
$$

The process is shown in figure 7 for the first sampling and correlation position, followed by a $\Delta$-shifted new one.

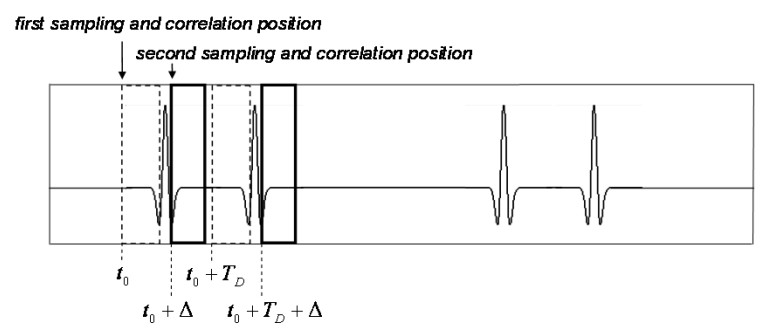

Figure 7: Time shift $\Delta$ between consecutive sampling and correlation windows.
Once the highest correlation value along the symbol is identified and so its position, the detector can correctly acquire the transmitted pulses at such instant. Note that this process is simplified compared to others detection methods, because it only needs that the UWB pulses fall inside the sampling windows $\mathrm{T}_{\mathrm{W}}$, in order to be reliably detected (Muller et al, 2007).

Figure 8 shows one example of the simulations made to test the synchronization procedure. The baseband TR-UWB signal, shown in 8.a, is composed of a sequence of symbols dedicated to the synchronization and some subsequent symbols that carry on information bits modulated with a phase shift of $180^{\circ}$. In figure $8 . \mathrm{b}$ it is shown the signal translated in frequency at $4 \mathrm{GHz}$ with the effects of the channel and added noise. The last graphic shows the results of the correlations made to obtain the synchronization and to detect the information bits. In this example the maximum value is obtained for $k=$ 15 , which means that the doublet is located $15 \Delta$ seconds after the starting reference time $t_{0}$.
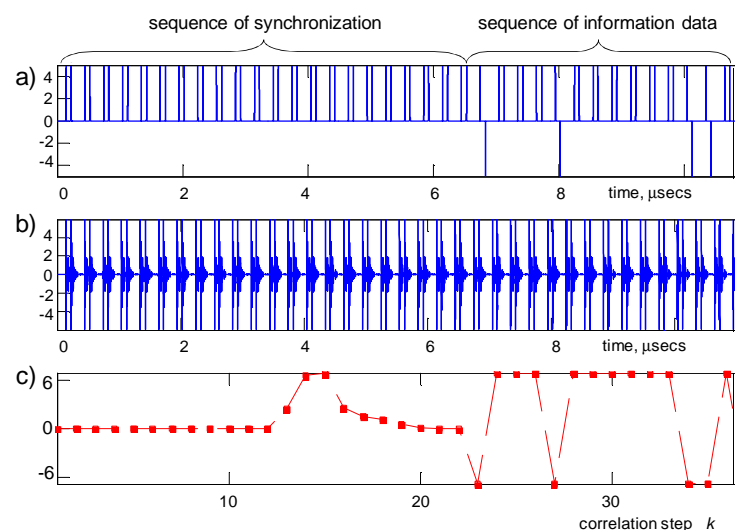

Figure 8: Correlation values obtained after the swept of the equivalent of one complete symbol.

In the simulations it was calculated one point of correlation for each symbol, meaning that $\mathrm{K}$ symbols are necessary to obtain the whole swept equivalent to the time duration of one symbol.

The synchronization procedure is based on the detection of pulses during the synchronization sequence and once this is obtained then the detection of the information bits is carried out. The next section demonstrates the detection of the pulses of the TR-UWB signal using the TDSC circuit with different configurations. 


\section{EVALUATION TESTS AND RESULTS}

This section presents the tests carried out to validate the detection method from real signals acquired through the TDSC CMOS prototype. First the structure of the test cards is described, in order to show the conditions under which the validation was done. Second it is explained the different configurations used for the tests and finally the experimental results are presented.

\subsection{Test Cards}

In order to specify the chip performance and validate for the first time the detection method, the very first basic tests of the TDSC IC were done over a test board using a generator instrument and a digital oscilloscope (Hirata-Flores, 2008). For a broader test of the circuit and the evaluation of the synchronization method and detection of data presented in this paper, two test cards were designed and realized: a transmitter card and a receiver card.

The transmitter card is based on a DS89C450 microcontroller to produce a squared signal that generates the frame, a step recovery diode (SRD) for producing very short-in-time pulses and a shortcircuit line to help produce the final waveform. Thanks to this transmitter, TR-UWB signals are generated in a simple way independently of other equipment. It is also possible to control the time between the pulses that could be as short as $140 \mathrm{~ns}$ and can be incremented in 33 ns steps. Figure 9 shows the block diagram of the transmitter.

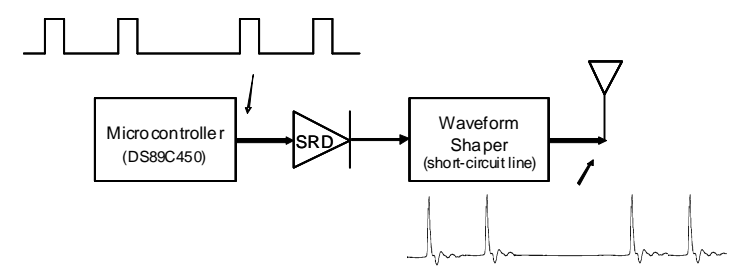

Figure 9: Block diagram and pulses waveforms of the transmitter test card.

The receiver card has been designed to help the development and the evaluation of the detection and synchronization methods. The card is also based on the DS89C450 microcontroller, which sends the start control signals to the TDSC circuit, as those shown in figure 5, to sample and write into the registers the incoming TR-UWB signal. The outputs of the registers are fed to an analog to digital converter
(ADC) and sent to a computer for further processing through a MATLAB program as shown in figure 10 . The signal detection is done by correlating the acquired signals. All the control signals to the TDSC circuit, the converters and the RS232 interface are generated by the microcontroller.

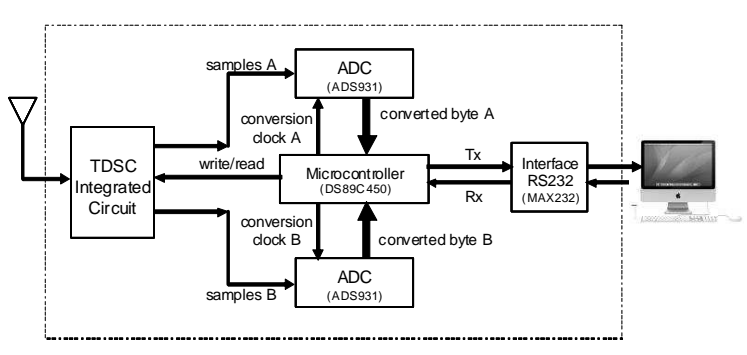

Figure 10: Block diagram of the receiver test card.

\subsection{Test Configuration}

The tests were run using two kinds of TR-UWB signals: a baseband signal, whose bandwidth extends to around $1 \mathrm{GHz}$, and a frequency translated signal centred at $4 \mathrm{GHz}$. It was used both a wired and a real wireless channel.

For the tests using a wired channel, both the transmitter and the receiver were connected directly by a cable as shown in figure 11 .

a)

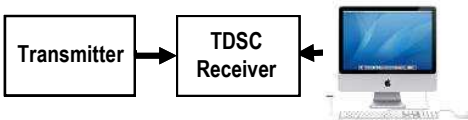

b)

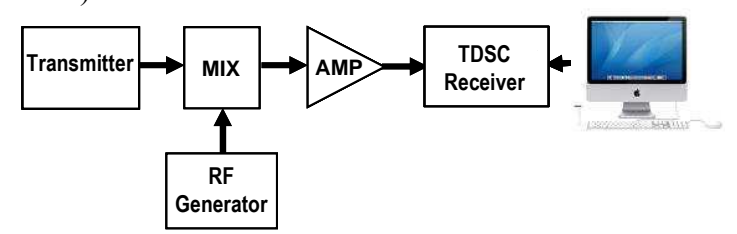

Figure 11: a) Direct connection between test cards at base band. b) Transmitted signal translated at $4 \mathrm{GHz}$.

In the case of the tests at $4 \mathrm{GHz}$, a mixer was employed to easily translate the baseband signal in frequency, also shown in figure 11.

In the case of tests with a wireless channel, both the baseband signal and the $4 \mathrm{GHz}$ signal were transmitted through antennas (monopole and discones), although these were not optimized for the system. Figure 12 shows the configurations used. 
a)

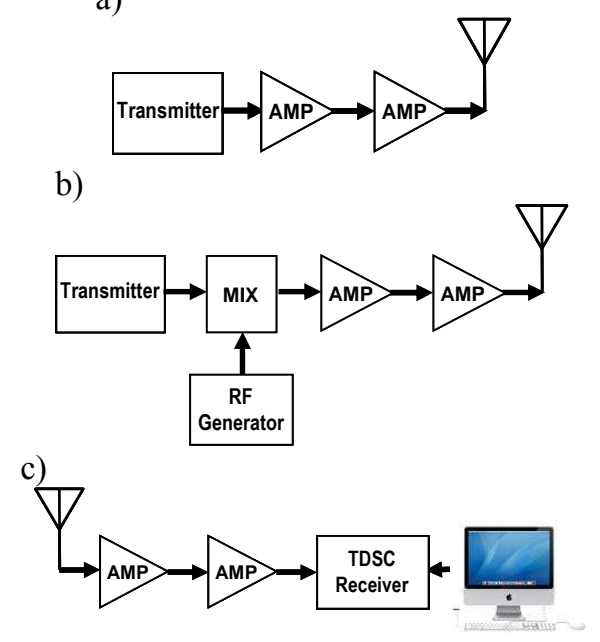

Figure 12: a) Transmitter configuration used at base band. b) Transmitter configuration used at $4 \mathrm{GHz}$. c) Receiver configurations for both cases.

\subsection{Experimental Results}

\subsubsection{Transmitted Signal}

The transmitted TR-UWB signal comprised pulses with a length of around $1 \mathrm{~ns}$, a $T_{D}$ spacing time of $366 \mathrm{~ns}$ and a symbol period of 2.66 us. The waveform reaches a maximum amplitude of 1.16 volts as shown in figure 13.

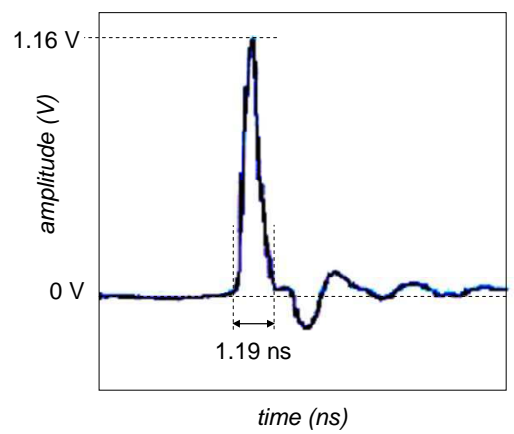

Figure 13: Waveform of the transmitter output pulse.

In figure 14 it is shown the power spectral density of the signal measured directly at the output of the transmitter. As we can see, the bandwidth at the power level of $-43 \mathrm{dBm}$ reaches a value of around $1.1 \mathrm{GHz}$. The sampling frequency of the
TDSC circuit that was used during the tests was estimated equal to $7.5 \mathrm{GHz}$ (Hirata-Flores, 2008).

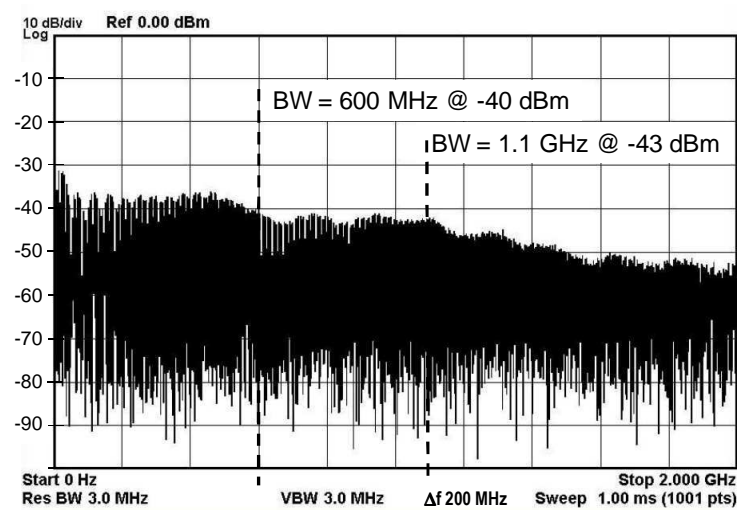

Figure 14: Power spectrum density of the transmitter output pulse.

To allow the MATLAB program to automatically manage the reception process, it was necessary to previously manually calibrate it. This was achieved for each test case, identifying an adequate threshold value of the correlation to distinguish between the presence and absence of pulses in the received signal. In this way the test system was able to continually receive and detect the symbols in a reproducible way. The figures presented in the following subsection correspond to one example of the received signals for each case.

\subsubsection{Configuration with Cable Connection}

Figures 15 and 16 show the waveforms received in the case of the direct connection between the transmitter and the receiver. The former corresponds to the baseband signal, while the latter is the signal translated at $4 \mathrm{GHz}$. The first and the second waveforms are the contents of registers A and B respectively, while the last waveform is the result of the cross-correlation function between the two of them. The value shown as "Corr. Max" corresponds to the maximum value of the cross-correlation, $c_{W 0}$, as it was explained in section 2.2 and defined in (8). The amplitude of the signals is normalized.

In both cases, baseband and pass band at $4 \mathrm{GHz}$, it was successfully found and fixed a threshold for the correlation value to detect the pulses. While for the baseband the maximum correlation value was around 4.21 , for the pass band signal it was around 0.2 . 

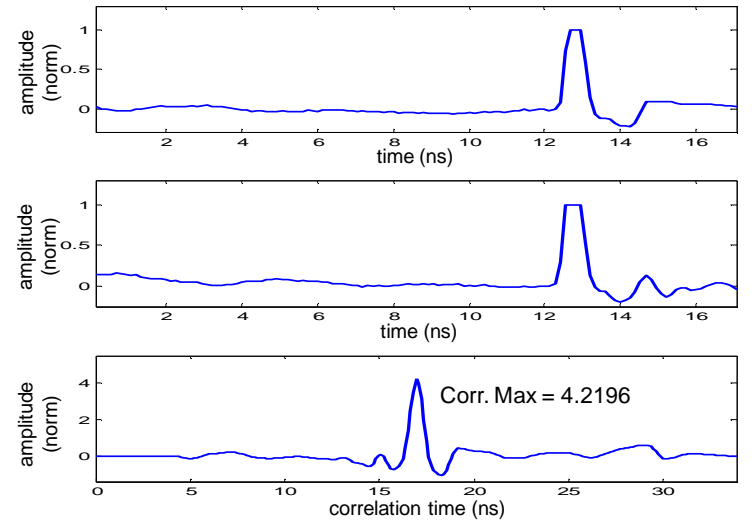

Figure 15: Results with a baseband signal directly connected to the receiver.
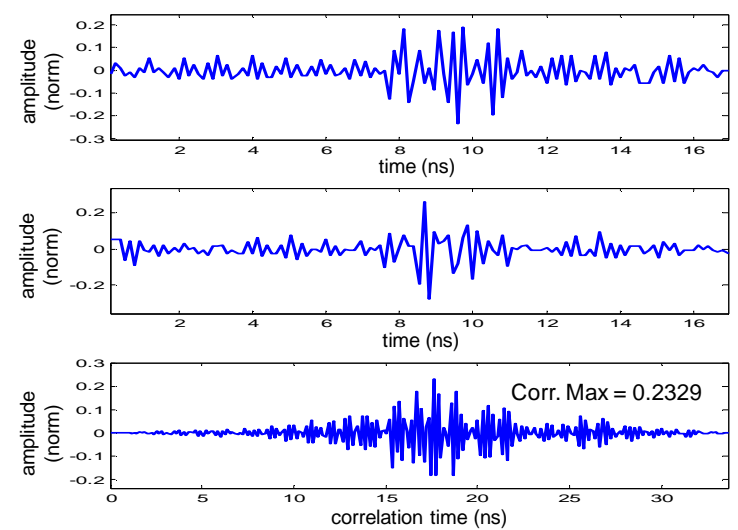

Figure 16: Results with a $4 \mathrm{GHz}$ signal directly connected to the receiver.

\subsubsection{Configuration with Wireless Channel}

Figure 17 shows the results obtained for a baseband signal transmitted using a pair of monopole antennas separated by $40 \mathrm{cms}$ between them. When the pulses are present in the TDSC circuit registers, the maximum correlation value is around 0.21 .

Figure 18 shows the results for the transmission of a baseband signal whose transmitting point is located at a distance of one meter from the receiver. In this case the value that shows that the two pulses were detected is between 0.14 and 0.135 .
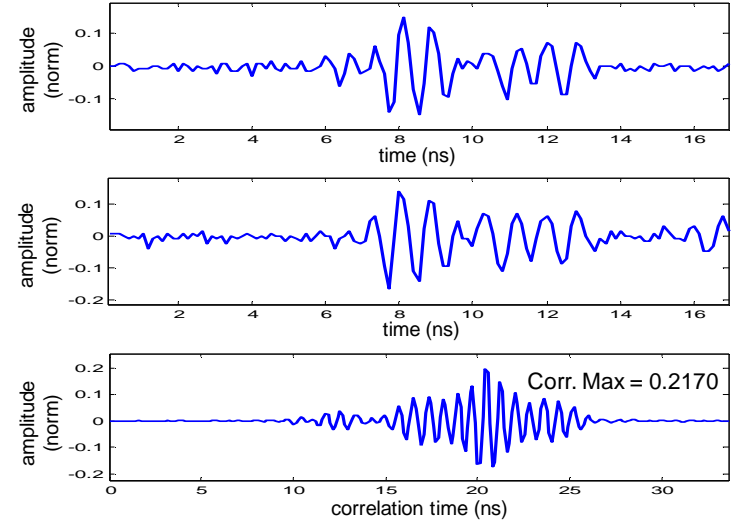

Figure 17: Results with a baseband signal transmitted at $40 \mathrm{cms}$. with line of sight (LOS).
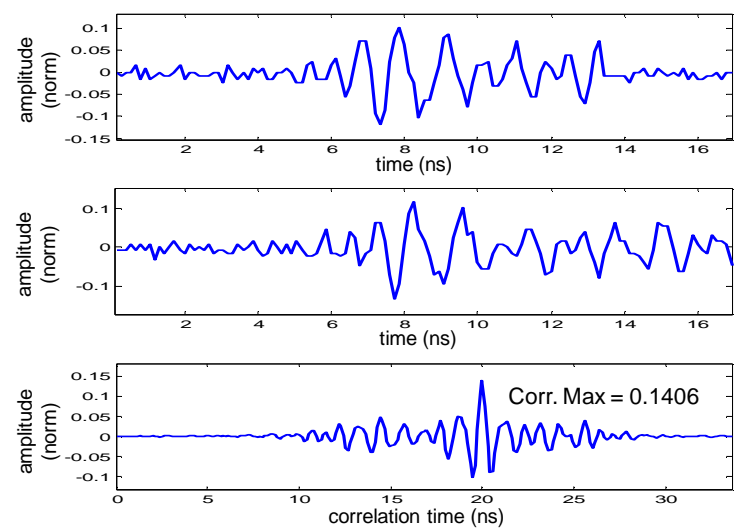

Figure 18: Results with a baseband signal transmitted at $100 \mathrm{cms}$. with line of sight (LOS).

In order to allow comparison between the maximum correlation values, figure 19 shows the results according to a signal without transmitted pulses, while figure 20 shows the case with only one baseband pulse transmitted at $40 \mathrm{cms}$. The first case gives a correlation value of 0.0093 , while in the second case the value obtained is 0.022 , both values are far enough of the value corresponding to the detection of the two pulses at the same distance, around 0.21 as previously stated. 

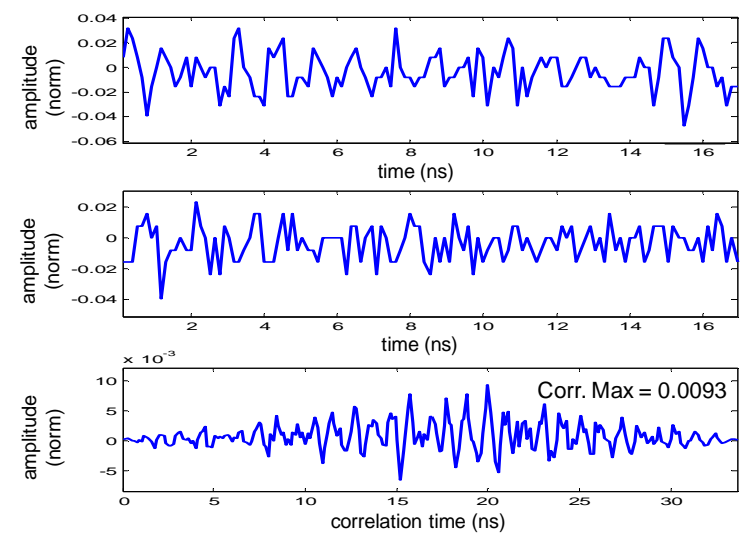

Figure 19: Results without transmitted pulses.
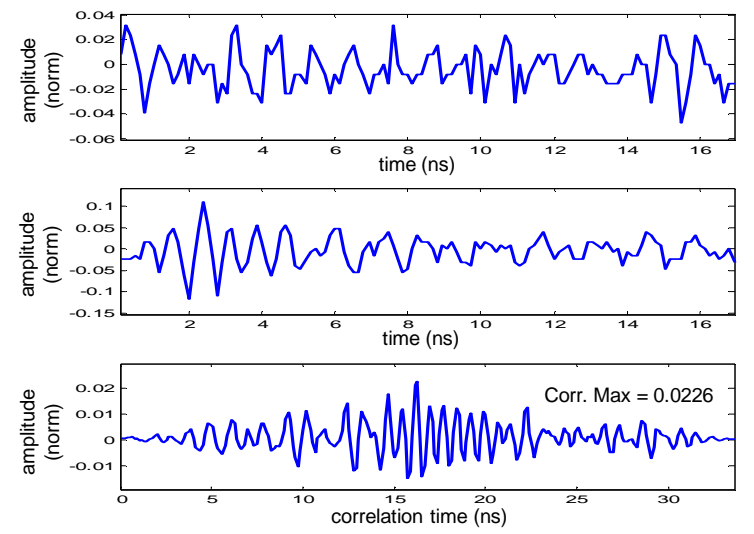

Figure 20: Results with only one pulse.

The last case shown in figure 21 corresponds to a pass band signal transmitted at $4 \mathrm{GHz}$ by a pair of discone antennas. The distance between the transmitter and the receiver is $40 \mathrm{cms}$. The pulses were successfully received for a corresponding correlation value of about 0.12 .
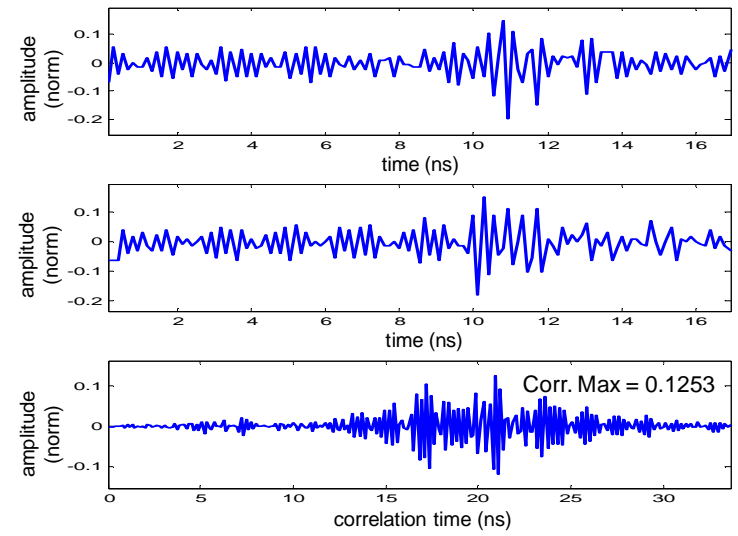

Figure 21: Results with a $4 \mathrm{GHz}$ signal transmitted at 40 cms. with line of sight.
These results show that the proposed detection method can successfully detect TR-UWB signals through the TDSC CMOS prototype. Good detection results were obtained for different environments and distances. Being the detection the base of the synchronization process, these tests have also validated the first stage of the synchronization process.

\section{CONCLUSIONS}

This paper has presented experimental results that validate the new TDSC detection method for TR-UWB systems using real signals.

Through this work we also improve the knowledge of the TDSC performance regarding the $\mathrm{T}_{\mathrm{D}}$ implementation, frequency response, signal detection through indoor wireless channel.

In order to obtain these results, two test cards were developed. One is a TR-UWB transmitter based on discrete components, the second one is the receiver based on the CMOS TDSC chip and connected to the computer.

The processing step of the proposed detection method based on the analog waveforms correlation was implemented on the computer.

The analog TR-UWB signals were acquired from a directed connection as well through an indoor wireless channel to a distance as long as 1 meter as first step of experimentation.

It was demonstrated that the use of the TDSC integrated circuit, as the receiving device, allows the detection of such signals through different environment or frequency condition (BB or $4 \mathrm{GHz}$ ). That validates also the generation of a precise delay $\mathrm{T}_{\mathrm{D}}$ in order to detect TR-UWB signals.

From these results the next steps are to study other transmitting scenarios, for different environments and also to look forward for the implementation of the synchronization process into an autonomous device. This will allow the use of this platform for purposes of localization, which is the application subject of interest of the research team.

\section{REFERENCES}

ECMA International (2008). High rate ultra wideband PHY and MAC standard. ECMA-368, $3^{\text {rd }}$ edition.

F.C.C. (2002). Federal Communications Commission. Revision of part 15 of the commission's rules 
regarding ultra-wideband transmission systems. Technical report.

Gezici, S. (2008). A survey on wireless position estimation. Wireless Personal Communications, 44:263-282, 2008. 10.1007/s11277-007-9375-z.

Gezici, S. and Tian, Zhi and Giannakis, G.B. and Kobayashi, H. and Molisch, A.F. and Poor, H.V. and Sahinoglu, Z. (2005, July). Localization via ultrawideband radios: a look at positioning aspects for future sensor networks. Signal Processing Magazine, IEEE, 22(4):70 - 84.

Hirata-Flores, F.I. and Muller, M. and Ni, Yang and Gimenes, C. (2008). CMOS Implementation of a TRUWB Receiver Based on Time Delayed Sampling and Correlation Method. Wireless Conference, 2008. EW 2008. 14th European 22-25, Prague.

Hirata-Flores, F.I. (2008). Design and Implementation of a CMOS Prototype for a TDSC-UWB Receiver Based on TR Detection Scheme. PH.D 2008, Telecom SudParis, France.

IEEE. (2007). IEEE Std 802.15.4a TM-2007. IEEE standard for information technology - telecommunications and information exchange between systems - local and metropolitan area networks - specific requirement part 15.4: Wireless medium access control (mac) and physical layer (phy) specifications for low-rate wireless personal area networks (wpans).

Jofre, L. and Broquetas, A. and Romeu, J. and Blanch, S. and Toda, A.P. and Fabregas, X. and Cardama, A. (2009) UWB tomographic radar imaging of penetrable and impenetrable objects. Proceedings of the IEEE, 97(2):451 -464.

Molisch, A.F. and Cassioli, D. and Chong, Chia-Chin and Emami, S. Fort, A. and Kannan, B. and Karedal, J. and Kunisch, J. and Schantz, H.G. and Siwiak, K. and Win, M.Z. (2006). A comprehensive standardized model for ultrawideband propagation channels. Antennas and Propagation, IEEE Transactions on, 54(11):3151 -3166.

Muller, M. and Ni Y. and Lamberti R. and Hirata-Flores F.I. and Saber, C. (2007). Fully CMOS Low Power Low Complexity Detection Method For TR-UWB; FTFC 2007 Paris.

Muller, M. and Ni, Y. and Lamberti, R. and Saber, Ch. (2008) A new signal detection method for tr-uwb: By time delayed sampling and correlation (tdsc). In Joaquim Filipe and Mohammad S. Obaidat, editors, EBusiness and Telecommunication Networks, volume 9 of Communications in Computer and Information Science, pages 301-311. Springer Berlin Heidelberg.

Saber, Ch. (2008). Ultra Large Bande Radio par Impulsions. Contributions à la Définition du Récepteur TDSC. Relation à la filière technologique. PH. D 2008. Telecom SudParis, France.

Saber, Ch. and Lamberti, R. and Gimenes, C. (2008). Synchronization solution for the tdsc-uwb detection method. In Tarek Sobh, Khaled Elleithy, Ausif
Mahmood, and Mohammad A. Karim, editors, Novel Algorithms and Techniques In Telecommunications, Automation and Industrial Electronics, pages 311316. Springer Netherlands, 2008. 10.1007/978-14020-8737-0_56. 